Mon. Not. R. Astron. Soc. 000,19(2010) Printed Date Not Specified $\quad$ (MN LATEX style file v2.2)

\title{
Modified Virial Formulae and the Theory of Mass Estimators
}

\author{
J. $A n^{1}$ and N. W. Evans ${ }^{2}$ \\ ${ }^{1}$ National Astronomical Observatories, Chinese Academy of Sciences, A20 Datun Road, Chaoyang District, Beijing 100012, PR China; \\ ${ }^{2}$ Institute of Astronomy, University of Cambridge, Madingley Road, Cambridge CB3 OHA.
}

(TO APPEAR IN Monthly Notices of the Royal Astronomical Society)

\begin{abstract}
We show how to estimate the enclosed mass from the observed motions of an ensemble of test particles. Traditionally, this problem has been attacked through virial or projected mass estimators. Here, we examine and extend these systematically, and show how to construct an optimal estimator for any given assumption as to the potential. The estimators do not explicitly depend on any properties of the density of the test objects, which is desirable as in practice such information is dominated by selection effects. As particular examples, we also develop estimators tailored for the problem of estimating the mass of the Hernquist or NFW dark matter haloes from the projected positions and velocities of stars.
\end{abstract}

Key words: galaxies: general - galaxies: haloes - galaxies: kinematics and dynamics - galaxies: fundamental parameters - dark matter

\section{INTRODUCTION}

Here, we consider the general problem of estimating the enclosed mass (or equivalently the gravitational potential) from kinematical data on tracer populations. In other words, suppose there are $N$ test particles moving in a gravitational potential $\phi(\boldsymbol{r})$ generated by a mass density $\rho(\boldsymbol{r})$. The data available to us are the instantaneous positions $\boldsymbol{r}_{i}$ and velocities $\boldsymbol{v}_{i}$ of the test bodies. However, it is only rarely that the full phase space information is available and often only components of position and velocity along the line-of-sight are measured. From these data, we wish to estimate the underlying gravitational potential or mass by a robust and unbiased statistical method.

This problem has many applications in modern astrophysics - including estimating the mass of the Milky Way and M31 from the kinematics of distant satellites (Little \& Tremaine 1987; Wilkinson \& Evans 1999; Watkins et al. 2010), estimating the mass of the haloes of dwarf galaxies from the stellar velocities (Strigari et al. 2008; Walker et al. 2009; Wolf et al. 2010), and estimating the mass of galaxy groups and clusters from their members (Heisler et al. 1985; Tully et al. 2006). In fact, the kinematical properties of tracer populations are one of the richest sources of data on the distribution of dark matter in galaxies and clusters. Therefore, it is important to extract as much information from the data as we possibly can.

Given the significance of the problem, there has been surprisingly little effort on developing the systematic theory of mass estimators. Early work (Limber \& Mathews 1960) exploited the virial theorem to obtain

$M=\frac{3 \pi}{2 G} \frac{\left\langle v_{\ell}^{2}\right\rangle}{\left\langle R^{-1}\right\rangle} \approx \frac{3 \pi}{2 G} \frac{\sum_{i}^{N} v_{\ell, i}^{2}}{\sum_{i}^{N} R_{i}^{-1}}$

for the mass enclosed by $N$ test particles with line-of-sight veloc- ities $v_{\ell, i}$ and projected positions $R_{i}$ of each particle. The problem with this method was pointed out by Bahcall \& Tremaine (1981), namely that the virial mass estimator is both biased and inefficient. These latter authors introduced the alternative projected mass estimator

$M=\frac{C}{G}\left\langle v_{\ell}^{2} R\right\rangle \approx \frac{C}{G N} \sum_{i}^{N} v_{\ell, i}^{2} R_{i}$

where $C$ is a constant determined by the host potential and the eccentricity of the orbits.

There has also been substantial previous work done on scalefree mass estimators (White 1981; Kulessa \& Lynden-Bell 1992; Evans et al. 2003). Recently, Watkins, Evans \& An (2010) formalized and expanded the ideas from these previous papers, presenting a variety of mass estimators tailored to scale-free potentials and densities. These work by taking weighted averages of the combinations of velocities and positions that remain invariant under similarity transformations. None the less, it is also important to devise mass estimators that are optimized for more realistic and specific astrophysical potentials. For example, there are cosmological arguments that the dark halo density is cusped like $r^{-1}$ at small radii and falls off like $r^{-3}$ at large radii (Navarro, Frenk \& White 1995). It is natural to look for mass estimators that build upon this assumption at the very start.

In this paper, we show how to find such mass estimators tailored for any given potential. We also find that our new estimators do not depend on the number density of the tracers at all. This is a real advantage - e.g., in the case of the Milky Way, the variation of the number density of the known satellite galaxies and globular clusters is dominated by the selection effects and the true number density can only be guessed at.

This paper is arranged as follows. In Sect. 2, we develop some general theories on mass estimators, showing how to construct one 
suitable for given potential. In Sect. 3 we give a few specific examples for the cases of astrophysical interest, whilst Sect. 4 sketches the extension to projected data. The self-consistent case, when the potential and the density of the tracers are related through the Poisson equation, is dealt with in Sect.5 Finally, in Sect.6 we provide a discussion and conclusions. Some applications of our estimators to the widely-used cosmological halo model of Navarro et al. (1995) are found in Evans, An \& Deason (2010), which should be considered as a companion to the current paper.

\section{THE THEORY OF MASS ESTIMATORS}

\subsection{Jeans' Equation and the Virial Theorem}

Suppose our tracer population has a number density $v(r)$ and a radial velocity dispersion $\sigma_{r}^{2}(r)$ and is moving in a spherical dark halo potential $\phi(r)$, which by Newton's Theorem satisfies

$\frac{\mathrm{d} \phi}{\mathrm{d} r}=-\frac{G M(r)}{r^{2}}$

where $M(r)$ is the enclosed halo mass within radius $r$. These quantities are related to one another through the spherical Jeans equation that reads

$\frac{\mathrm{d}}{\mathrm{d} r}\left(v \sigma_{r}^{2}\right)+\frac{2 \beta}{r} v \sigma_{r}^{2}=v \frac{\mathrm{d} \phi}{\mathrm{d} r}$

where

$\beta=1-\frac{\sigma_{\theta}^{2}+\sigma_{\phi}^{2}}{2 \sigma_{r}^{2}}=1-\frac{\sigma_{\theta}^{2}}{\sigma_{r}^{2}}$

is the so-called Binney anisotropy parameter for the spherical system. The typical application of the Jeans equations involves deriving the potential and the dark halo mass profile from the observed behaviour of the tracer density and velocity dispersions ('Jeans modelling'). If the observations are composed of discrete sample datapoints, this is subject to the uncertainties related to the binning and requires large number of datapoints to extract any meaningful information. An alternative when only moderate number of datapoints are available is to consider the system as whole such as utilizing the virial theorem. The relation between these two is most obvious in a spherical system, for which integrating the spherical Jeans equation essentially results in the scalar virial theorem.

In order to see this, we start by noting that the spherical Jeans equation reduces to an exact differential form

$\frac{1}{Q} \frac{\mathrm{d}}{\mathrm{d} r}\left(Q v \sigma_{r}^{2}\right)=-v \frac{G M}{r^{2}}$

by means of the integrating factor $Q=Q(r)$ satisfying

$\frac{\mathrm{d} \ln Q(r)}{\mathrm{d} r}=\frac{2 \beta(r)}{r}$.

Next we find that the relation between the local three-dimensional velocity dispersion and the radial velocity dispersion is given by

$\sigma^{2}=\sigma_{r}^{2}+\sigma_{\theta}^{2}+\sigma_{\phi}^{2}=(3-2 \beta) \sigma_{r}^{2}=\sigma_{r}^{2} \frac{\mathrm{d} \ln \left(r^{3} Q^{-1}\right)}{\mathrm{d} \ln r}$.

The three-dimensional velocity dispersion of the tracers within the sphere of the radius of $r_{\text {out }}$ is thus given by

$\left\langle v^{2}\right\rangle=\frac{4 \pi}{N_{\text {tot }}} \int_{0}^{r_{\text {out }}} \mathrm{d} r r^{2} v \sigma^{2}=\frac{4 \pi}{N_{\text {tot }}} \int_{0}^{r_{\text {out }}} Q v \sigma_{r}^{2} \frac{\mathrm{d}\left(r^{3} Q^{-1}\right)}{\mathrm{d} r} \mathrm{~d} r$

where

$N_{\text {tot }}=4 \pi \int_{r_{\text {in }}}^{r_{\text {out }}} \mathrm{d} r r^{2} v$ is the total number of the tracers between an inner $r_{\text {in }}$ and outer $r_{\text {out }}$ radius. Integrating by parts, setting $r_{\text {in }}=0$ and also using equation (2) results in

$$
\begin{aligned}
\frac{N_{\text {tot }}}{4 \pi}\left\langle v^{2}\right\rangle & =\left.v \sigma_{r}^{2} r^{3}\right|_{0} ^{r_{\text {out }}}-\int_{0}^{r_{\text {out }}} \frac{r^{3}}{Q} \frac{\mathrm{d}\left(Q v \sigma_{r}^{2}\right)}{\mathrm{d} r} \mathrm{~d} r \\
& =\left.v \sigma_{r}^{2} r^{3}\right|_{r=r_{\text {out }}}+\int_{0}^{r_{\text {out }}} \mathrm{d} r G M r v,
\end{aligned}
$$

given that $\sigma_{r}^{2}$ is not divergent as $r \rightarrow 0$. Here the last integral actually defines the total potential energy of the tracers within the same sphere, i.e.,

$|W|=4 \pi \int_{0}^{r_{\text {out }}} \mathrm{d} r r^{2} v \frac{G M}{r} ; \quad\left\langle\frac{G M}{r}\right\rangle=\frac{|W|}{N_{\text {tot }}}$.

Hence, equation 5 reduces to

$\left\langle v^{2}\right\rangle=\left\langle\frac{G M}{r}\right\rangle+3 \varsigma^{2}$

where

$\varsigma^{2}=\frac{v\left(r_{\text {out }}\right) \sigma_{r}^{2}\left(r_{\text {out }}\right)}{\bar{v}_{\text {out }}} ; \quad \bar{v}_{\text {out }}=\frac{3 N_{\text {tot }}}{4 \pi r_{\text {out }}^{3}}$.

Note that $\bar{v}_{\text {out }}$ is the mean number density of the tracers in the sphere of the radius of $r_{\text {out }}$.

Equation (6) is in fact equivalent to the statement of the scalar virial theorem for a pressure-supported spherical system as $\frac{1}{2}\left\langle v^{2}\right\rangle$ is basically the kinetic energy per tracer particle associated with the random motion. The presence of the boundary term (that is, the surface term, $3 \varsigma^{2}$ ) is due to the hard cut-off at $r=r_{\text {out }}$, which can correspond to the situation when the tracers are confined within the spherical radius of $r_{\text {out }}$ through the external pressure. However, it is usual to drop the boundary term if the system as a whole is considered.

\subsection{Tracer Mass Estimators}

The virial theorem (eq. 6) is traditionally used to estimate the total mass of the system. The integral mean value theorem indicates that there exists a kind of 'mean radius' $\bar{r}$ within the interval bounded by the outer cut-off $r_{\text {out }}$ (i.e., $0<\bar{r} \leqslant r_{\text {out }}$ ) such that $\langle G M / r\rangle=G M(\bar{r}) / \bar{r}$. Therefore, if one ignores the boundary term, one can relate the mass within the 'mean radius' to the total velocity dispersion of the tracers, $M(\bar{r})=\bar{r}\left\langle v^{2}\right\rangle / G$, which may also be suspected from a simple dimensional analysis. If the distribution of the gravitating mass is known or assumed, the definition of $\bar{r}$ can be made precise and furthermore $M(\bar{r})$ can be scaled to provide the estimate of $M\left(r_{\text {out }}\right)$. That is to say, we can relate the total mass of the system to the certain integrals of kinetic properties of the tracers.

However, this approach suffers from drawbacks related to the fact that the mass estimate depends on two separate averages (see e.g., Bahcall \& Tremaine 1981). This difficulty is partially overcome by the use of 'mass estimator', that is, an average of particular combinations of kinetic properties of the tracers that directly relates to the total mass rather than to the potential energy, as does the virial estimator. However, derivation of the proper form of the mass estimator requires some analysis of the dynamics of the system. Here we still consider the simplest case of the spherical system traced by a non-rotating relaxed population in equilibrium.

First we note that the virial theorem (with the boundary term dropped) indicates that $\left\langle v^{2}\right\rangle /\left\langle v_{\mathrm{c}}^{2}\right\rangle=1$ where $v_{\mathrm{c}}=(G M / r)^{1 / 2}$ is the circular speed of the potential. From this, one may naively expect that $\left\langle v^{2} / v_{\mathrm{c}}^{2}\right\rangle \approx 1$, but the distributed mass and tracers only make this 
approximately so. However, with a proper weighting $f(r)$, we can actually show that there exists a relation $\left\langle f v_{r}^{2} / v_{\mathrm{c}}^{2}\right\rangle=1$, which will be subsequently used to derive a proper mass estimator.

Let us assume for the moment that the spherical dark halo profile $M(r)$ is known. Then we can show that the proper weighting function is given by

$$
\begin{aligned}
f(r) & =4-2 \beta(r)-\frac{\mathrm{d} \ln M(r)}{\mathrm{d} \ln r} \\
& =\frac{\mathrm{d} \ln \left(r^{4} Q^{-1} M^{-1}\right)}{\mathrm{d} \ln r}=\frac{Q M}{r^{3}} \frac{\mathrm{d}}{\mathrm{d} r}\left(\frac{r^{4}}{Q M}\right) .
\end{aligned}
$$

Then the 'weighted' average of the tracer radial velocities $v_{r}^{2}$ in a spherical system given by

$$
\begin{aligned}
\left\langle\frac{f v_{r}^{2}}{v_{\mathrm{c}}^{2}}\right\rangle & =\frac{4 \pi}{N_{\text {tot }}} \int_{r_{\text {in }}}^{r_{\text {out }}} \frac{f v \sigma_{r}^{2} r^{3}}{G M} \mathrm{~d} r \\
& =\frac{4 \pi}{G N_{\text {tot }}} \int_{r_{\text {in }}}^{r_{\text {out }}} Q v \sigma_{r}^{2} \frac{\mathrm{d}}{\mathrm{d} r}\left(\frac{r^{4}}{Q M}\right) \mathrm{d} r .
\end{aligned}
$$

Integrating by part leads to

$$
\begin{aligned}
\frac{G N_{\text {tot }}}{4 \pi}\left\langle\frac{f v_{r}^{2}}{v_{\mathrm{c}}^{2}}\right\rangle & =\left.\frac{v \sigma_{r}^{2} r^{4}}{M}\right|_{r_{\text {in }}} ^{r_{\text {out }}}-\int_{r_{\text {in }}}^{r_{\text {out }}} \frac{r^{4}}{Q M} \frac{\mathrm{d}}{\mathrm{d} r}\left(Q v \sigma_{r}^{2}\right) \mathrm{d} r \\
& =\left.\frac{v \sigma_{r}^{2} r^{4}}{M}\right|_{r_{\text {in }}} ^{r_{\text {out }}}+\frac{G N_{\text {tot }}}{4 \pi},
\end{aligned}
$$

where we have also used equation (2).

For any physical system, we apply the result of An \& Evans (2009) to find that $\lim _{r \rightarrow 0} r^{4} v \sigma_{r}^{2} / M=01$ Hence, setting $r_{\text {in }}=0$ leads to the vanishing inner boundary term, and thus

$\left\langle\frac{f v_{r}^{2}}{v_{\mathrm{c}}^{2}}\right\rangle=1+3 \frac{\varsigma^{2}}{v_{\mathrm{c}}^{2}\left(r_{\text {out }}\right)}$.

This relation can be rearranged to yield the total dark halo mass $M_{\text {out }}=M\left(r_{\text {out }}\right)$ within radius $r_{\text {out }}$,

$G M_{\text {out }}=\left\langle\frac{f r v_{r}^{2}}{\tilde{\mu}}\right\rangle-3 r_{\mathrm{out}} \varsigma^{2}$,

where

$\tilde{\mu}(r)=\frac{M(r)}{M_{\text {out }}} \quad\left(0 \leqslant r \leqslant r_{\text {out }}\right)$

is the normalized dark halo mass profile function. Note that $\mathrm{d} \ln \tilde{\mu} / \mathrm{d} \ln r=\mathrm{d} \ln M / \mathrm{d} \ln r$, and thus $f(r)$ can be evaluated if $\tilde{\mu}(r)$ is known without any reference to $M_{\text {out }}$. Consequently, if we are willing to assume the functional form of the halo mass profile $\tilde{\mu}(r)$, which is normalized to be $\tilde{\mu}\left(r_{\text {out }}\right)=1$, in a spherical region of interest, $r<r_{\text {out }}$, then the total halo mass $M_{\text {out }}$ within the same spherical region can be estimated through a particular average of kinematic

\footnotetext{
1 Naively, we have $\lim _{r \rightarrow 0} r^{3} v=0$ for any physical $v$ since otherwise there would be an infinite mass concentration of the tracers at the centre whereas $\lim _{r \rightarrow 0} r \sigma_{r}^{2} / M$ is typically finite An \& Evans 2009). More careful examination of An \& Evans (2009) indicates that even for the exceptional case that $\lim _{r \rightarrow 0} r \sigma_{r}^{2} / M$ diverges, the boundary term still vanishes as $\lim _{r \rightarrow 0} r^{3} v=0$ is always dominant. That is to say, we infer from An \& Evans (2009) that, given $M(r)$ behaving as $\sim r^{1-\alpha}$ as $r \rightarrow 0$, $\alpha>2 \beta-3$ is the sufficient condition for this. However, if $v \sim r^{-\gamma}$ as $r \rightarrow 0$, we have $2 \beta \leqslant \gamma \leqslant \alpha+2-$ the first inequality is due to An \& Evans (2006) and the second to the fact that the tracers cannot be cusped steeper than the dark halo - and so it is met.
}

properties of the tracers, which is in practice inferred from the corresponding discrete sample mean, i.e.,

$M_{\mathrm{out}} \approx \frac{1}{G N} \sum_{i}^{N} \frac{r_{i} f\left(r_{i}\right)}{\tilde{\mu}\left(r_{i}\right)} v_{r, i}^{2}$,

possibly further adjusted by the boundary term $\left(3 r_{\text {out }} G^{-1} \varsigma^{2}\right)$ if necessary.

Here, the presence of the boundary term is again related to the external pressure support of the tracer population. If the tracer population is a true isolated system of a finite spherical extent of $r_{\text {out }}$ in equilibrium with the dark halo potential, it follows that $\sigma_{r}^{2}\left(r_{\text {out }}\right)=v\left(r_{\text {out }}\right)=0$ and the boundary term naturally vanishes. A similar argument extends to the system of an infinite-extent tracer population with a finite-total-mass dark halo, for which $r_{\text {out }}=\infty$ also leads to the dropped outer boundary term (then $M_{\text {out }}=M_{\text {tot }}$ is now the 'true' total halo mass). However, if the tracer population is pressure-confined and/or the distribution of the observed tracers are truncated at a finite outer radius $r_{\text {out }}$, the outer boundary term must remain. In this case, if enough datapoints are available, the boundary term may be directly calculated from the observed tracer distribution.

\section{EXAMPLES}

We now develop formulae specific to some simple and widely-used halo models.

\subsection{The Scale-Free Potential}

The simplest mass model that we consider is $\left(0 \leqslant r \leqslant r_{\text {out }}\right)$

$\tilde{\mu}(r)=\left(\frac{r}{r_{\text {out }}}\right)^{1-\alpha}$

Here, $\alpha \leqslant 1$ is the power-index for the scale-free potential, or equivalently the rotation curve is given by

$v_{\mathrm{c}}^{2}(r)=\frac{r_{\text {out }}^{\alpha}}{r^{\alpha}} v_{\mathrm{c}}^{2}\left(r_{\text {out }}\right)$.

The central point-mass case is included with $\alpha=1$. If we require that the halo density does not increase outwards, then the index is restricted to be $\alpha \geqslant-2$, with $\alpha=-2$ corresponding to a homogeneous sphere.

For these cases, we have $f(r)=3-2 \beta+\alpha$. If $\beta$ is further assumed to be constant, then $f$ is also constant, and therefore equation (11) reduces to

$\frac{G M_{\text {out }}}{r_{\text {out }}}=(\alpha+3-2 \beta) \frac{\left\langle v_{r}^{2} r^{\alpha}\right\rangle}{r_{\text {out }}^{\alpha}}-3 \varsigma^{2}$

These are similar to the estimators used by Watkins et al. (2010) but the multiplicative coefficient $\alpha+\gamma-2 \beta$ in their equation (15) is replaced by $\gamma \Rightarrow 3$. This is because Watkins et al. (2010) further assumed a power-law behaviour for the tracer density profile, i.e., $v \propto r^{-\gamma}$, which allowed them to solve for $N_{\text {tot }}$ and $\sigma_{r}^{2}(r)$. Instead of dropping the boundary term by sending $r_{\text {out }} \rightarrow \infty$, which is an improper thing to do in a scale-free system, they equated $4 \pi r^{3} v / N(r) \approx 3-\gamma$ and $\sigma_{r}^{2} \simeq(\gamma-2 \beta+\alpha)^{-1} G M / r$ based on the power-law solutions. If we substitute these for the boundary term in equation (15), we recover their equation (15).

Among the scale-free potential cases, of particular interest are the Kepler $(\alpha=1)$ and the logarithmic $(\alpha=0)$ potential. For the 
Kepler potential generated by a central point mass, it is usual to set $r_{\text {out }}=\infty$ and $\varsigma^{2}=0$. Given that $\beta$ is also constant, this leads to

$\left\langle v_{r}^{2} r\right\rangle=\frac{G M \cdot}{2(2-\beta)}$,

where $M_{\bullet}$ is now the mass of the central point. On the other hand, for the logarithmic potential generated by a (truncated) singular isothermal sphere, equation 15 reduces to

$v_{\mathrm{c}}^{2}=\left\langle v^{2}\right\rangle-3 \varsigma^{2}$,

which is actually the same as equation (6) with $\langle G M / r\rangle=v_{\mathrm{c}}^{2}$ being constant.

\subsection{The Double-Power Law Halo}

A widely-used family to fit the simulated dark halo density profiles is in the form of $\rho(r) \propto r^{-a}\left(r_{0}^{p}+r^{p}\right)^{-(b-a) / p}$. If $b>3$, the total dark halo mass $M_{\text {tot }}$ is finite and $\tilde{\mu}(r)$ reduces to the regularized beta function with $r_{\text {out }}=\infty$. However, for many specific cases the results are much simpler. For example, if $[\rho(r)]^{-1} \propto r^{\gamma}\left(r_{0}^{p}+r^{p}\right)^{(3-\gamma) / p+1}$ with $0 \leqslant \gamma<3$ and $p>0$, then

$\frac{M(r)}{M_{\mathrm{tot}}}=\left(1+\frac{r_{0}^{p}}{r^{p}}\right)^{-(3-\gamma) / p} ; f=4-2 \beta-\frac{3-\gamma}{\left(r / r_{0}\right)^{p}+1}$.

That is, the functions $\tilde{\mu}(r)$ and $f(r)$ are in easily tractable analytic form provided that $\beta(r)$ is as such. This particular example includes the well-known families of the $\gamma$-sphere (Dehnen 1993; Tremaine et al. 1994) with $p=1$ and that of Veltmann (1979) and Evans \& An (2005) with $\gamma=2-p$.

In practice, the simple analytic form of $\tilde{\mu}(r)$ and $f(r)$ indicates that the sample mean of $\tilde{\mu}^{-1} f r v_{r}^{2}$ is straightforward to calculate for a fixed set of parameters. Moreover, provided that the tracer population extends sufficiently far out (that is, $r_{\text {out }} \gg r_{0}$ ) and so $M_{\text {out }} \equiv M\left(r_{\text {out }}\right) \approx M_{\text {tot }}$, we also argue that it is in general safe to drop the boundary term (formally $r_{\text {out }} \rightarrow \infty$, and $\tilde{\mu}=M / M_{\text {tot }}$ ).

For instance, for the Hernquist (1990) halo profile, that is, $p=$ $\gamma=1$ in equation (17), we find that

$$
\begin{aligned}
G M_{\mathrm{tot}} & =\left\langle\left(4-2 \beta-\frac{2}{1+r / r_{0}}\right)\left(1+\frac{r_{0}}{r}\right)^{2} r v_{r}^{2}\right\rangle \\
& \approx \frac{2 r_{0}^{2}}{N} \sum_{i}^{N}\left[1+\frac{2 r_{i}}{r_{0}}-\left(1+\frac{r_{i}}{r_{0}}\right) \beta_{i}\right]\left(1+\frac{r_{i}}{r_{0}}\right) \frac{v_{r, i}^{2}}{r_{i}}
\end{aligned}
$$

where $\beta_{i}=\beta\left(r_{i}\right)$. If $r_{i} \ll r_{0}$, the observable combination contributes like $\sim 2\left(1-\beta_{i}\right) r_{0}^{2} v_{r, i}^{2} r_{i}^{-1}$ whereas for $r_{i} \gg r_{0}$ the contribution is like $\sim 2\left(2-\beta_{i}\right) v_{r, i}^{2} r_{i}$, which is consistent with the local behaviour of the potential at the location of the tracer particle. we also note that the mass estimate is dependent upon the choice of the scale length $r_{0}$, which is expected.

\subsection{The NFW Halo}

The formal NFW profile (Navarro et al. 1995), i.e., $\rho^{-1} \propto r\left(r_{0}+r\right)^{2}$, on the other hand possesses infinite total mass, and the proper application of our scheme calls for the truncation of the profile either at the radius of the outermost tracer point $r_{\text {out }}$, or at the virial radius $r_{\mathrm{v}}$. Let us suppose that the tracers are well-populated so that the mass up to the virial radius $M_{\mathrm{v}} \equiv M\left(r_{\mathrm{v}}\right)$, i.e., the virial mass can be effectively estimated. Since $M(r) \propto m\left(r / r_{0}\right)$ where

$m(x)=\ln (1+x)-\frac{x}{1+x}$ for the NFW profile, if we let $M_{\text {out }}=M_{\mathrm{v}}$ and $r_{\text {out }}=r_{\mathrm{v}}$, then

$\tilde{\mu}(r)=\frac{m(\tilde{r})}{m(c)} ; \quad f(r)=4-2 \beta-\frac{1}{m(\tilde{r})}\left(\frac{\tilde{r}}{1+\tilde{r}}\right)^{2}$

where $c=r_{\mathrm{v}} / r_{0}$ is the concentration parameter and $\tilde{r}=r / r_{0}=$ $c r / r_{\mathrm{v}}$. For a fixed $r_{0}$, equation 11 is scaled to

$\frac{G M_{\text {out }}}{r_{0} m(c)}=\left\langle v_{r}^{2} \tilde{h}(\tilde{r})\right\rangle-\frac{3 c}{m(c)} \varsigma^{2}$

where $\tilde{h}(\tilde{r}) \equiv \tilde{r} f / m(\tilde{r})$ whose $r$-dependence is only via the scaled radius $\tilde{r}$. That is to say, the $c$-dependence of the result is essentially through the overall scale. Here, the boundary term is expected to be smal 2 and so it is all right to ignore it at the given level of the precision.

\section{MASS ESTIMATORS FOR PROJECTED DATA}

\subsection{Line-of-Sight Velocity Data}

In many situations, the radial velocities $\left(v_{r}\right)$ - with respect to the centre of the halo - of the tracers are not direct observables, but the line-of-sight velocities $\left(v_{\ell}\right)$ are. Fortunately, the adjustment of the estimator relating to the alternative velocity projections is straightforward. We use the relationship between the line-of-sight velocity dispersion $\left(\sigma_{\ell}\right)$ and the radial one $\left(\sigma_{r}\right)$;

$\sigma_{\ell}^{2}=\left(1-\beta \sin ^{2} \varphi\right) \sigma_{r}^{2}$

where $\varphi$ is the angle between the line of sight towards the tracer and the radial position vector of the same tracer from the centre of the halo. If the halo is sufficiently far away from us, each line of sight towards the individual tracer star or satellite galaxy runs approximately parallel to the line of sight towards the halo centre. Then, the angle $\varphi$ is equivalent to the spherical polar angular coordinate $\theta$ centred on the halo centre. In a spherical system, $\sigma_{r}^{2}$ is only dependent on $r$ and thus by averaging $\sigma_{\ell}^{2}$ over the angle $\theta \approx \varphi$ at a fixed $r$, we find that

$\int_{0}^{\pi / 2} \mathrm{~d} \varphi \sin \varphi\left(1-\beta \sin ^{2} \varphi\right) \sigma_{r}^{2}=\left[1-\frac{2}{3} \beta(r)\right] \sigma_{r}^{2}$.

Hence the weighted averages of $v_{\ell}^{2}$ and $v_{r}^{2}$ are related to each other such that

$\left\langle\frac{3 v_{\ell}^{2} h(r)}{3-2 \beta}\right\rangle=\left\langle v_{r}^{2} h(r)\right\rangle$

for any radial weighting function $h(r)$. This is valid for any $\beta(r)$ provided that the spherical symmetry assumption holds. For the constant $\beta$ cases, the factor $\left(1-\frac{2}{3} \beta\right)$ is a simple multiplicative constant that can be applied after the averaging.

Finally, the proper form of the mass estimator involving the line-of-sight velocities is obtained after substituting equation (21) into equation (11),

$\frac{G M_{\text {out }}}{3}=\left\langle\frac{3-2 \beta+\hat{\alpha}}{3-2 \beta} \frac{v_{\ell}^{2} r}{\tilde{\mu}}\right\rangle-r_{\text {out }} \varsigma^{2}$

where $\hat{\alpha}(r) \equiv 1-(\mathrm{d} \ln \tilde{\mu} / \mathrm{d} \ln r)$, which would be a constant $\hat{\alpha}=\alpha$ if $\tilde{\mu}$ is given by equation 14 .

2 For a virialized system, it is expected that $\sigma_{r}^{2}\left(r_{\mathrm{v}}\right) \approx 0$. In addition, if $r_{\mathrm{v}}=$ $r_{200}$, then $4 \pi r_{\mathrm{v}}^{3} v\left(r_{\mathrm{v}}\right) / N_{\text {tot }} \leqslant 4 \pi r_{\mathrm{v}}^{3} \rho\left(r_{\mathrm{v}}\right) / M_{\text {out }} \leqslant 3 / 200$ for the tracers that are more centrally concentrated than the dark halo. With typical concentration parameter values, then $3 \varsigma^{2} c / m(c) \lesssim 0.1 \sigma_{r}^{2}\left(r_{\mathrm{v}}\right)$. 


\subsection{Projected Separation Data}

In more typical cases for an external dark halo, we may only have the line-of-sight velocities and the projected distance $(R=r \sin \theta)$ to the halo centre known to within a reasonable precision. Here, we would like to find the proper weighting function $w(R)$ of $R$ such that $\left\langle v_{\ell}^{2} w(R)\right\rangle=\left\langle\tilde{\mu}^{-1} f r v_{r}^{2}\right\rangle$, which would replace equation 11 with

$G M_{\text {out }}=\left\langle v_{\ell}^{2} w(R)\right\rangle-3 r_{\text {out }} \varsigma^{2}$,

and lead to the projected mass estimator

$M_{\mathrm{out}} \approx \frac{1}{G N} \sum_{i}^{N} w\left(R_{i}\right) v_{\ell, i}^{2}$.

In practice, the average here would be over the cylindrical region with $R \leqslant R_{\text {out }}$ whereas the one in equation (11) is over the spherical region of $r \leqslant r_{\text {out }}$. The distinction is moot if the average is in fact over the whole space, i.e., $r_{\text {out }}=R_{\text {out }}=\infty$. With a finite cut-off radius $R_{\text {out }}$ in the tracer population, we proceed by assuming the true three-dimensional distribution of tracers is spherically symmetric and also cuts off at $r_{\text {out }}=R_{\text {out }}$. That is to say, the observed sample mean of $\left\langle v_{\ell}^{2} w(R)\right\rangle$ is considered to contain no contribution from tracers with $r>r_{\text {out }}$ and therefore to be a practical estimator for the average in the sphere of radius $r_{\text {out }}$.

Provided that the system is spherical and both averages are over the sphere, the condition that $\left\langle v_{\ell}^{2} w(R)\right\rangle=\left\langle v_{r}^{2} h(r)\right\rangle$ results in a integral equation for $w(R)$ at a fixed $r$;

$$
\begin{aligned}
r h(r) & =r \int_{0}^{\pi / 2} \mathrm{~d} \theta \sin \theta\left(1-\beta \sin ^{2} \theta\right) w(r \sin \theta) \\
& =\int_{0}^{r}\left[1-\beta(r) \frac{R^{2}}{r^{2}}\right] \frac{w(R) R \mathrm{~d} R}{\left(r^{2}-R^{2}\right)^{1 / 2}} .
\end{aligned}
$$

For $h=r f / \tilde{\mu}$, this is in principle invertible for $w(R)$ if $\lim _{r \rightarrow 0}(\mathrm{~d} \ln \tilde{\mu} / \mathrm{d} \ln r)<3$ and $\beta(r)$ is finite. We refer the reader to Appendix $\mathrm{A}$ for details.

In particular, if $\beta$ is a finite constant and $\tilde{\mu}$ is given by the scale-free form in equation (14) with $-2<\alpha \leqslant 1$, we find that

$w(R)=\frac{R^{\alpha}}{\hat{I}_{\alpha, \beta} r_{\text {out }}^{\alpha-1}}$

and

$\hat{I}_{\alpha, \beta}=\frac{\pi^{1 / 2} \Gamma\left(\frac{\alpha}{2}+1\right)}{4 \Gamma\left(\frac{\alpha+5}{2}\right)} \frac{\alpha+3-(\alpha+2) \beta}{\alpha+3-2 \beta}$

where $\Gamma(x)$ is the gamma function (the generalized factorial). That is to say, the corresponding mass estimator is in the form of (c.f., Watkins et al. 2010, eqs. 26 \& 27)

$\frac{G M_{\text {out }}}{r_{\text {out }}}=\frac{\left\langle v_{\ell}^{2} R^{\alpha}\right\rangle}{\hat{I}_{\alpha, \beta} r_{\text {out }}^{\alpha}}-3 \varsigma^{2}$.

Bahcall \& Tremaine (1981) considered the projected mass estimator for the central point-mass case. Their results are consistent with equation 25 with $\alpha=1$ once we drop the boundary term by setting $r_{\text {out }}=\infty$, that is,

$G M_{\bullet}=\frac{32}{\pi} \frac{2-\beta}{4-3 \beta}\left\langle v_{\ell}^{2} R\right\rangle$.

Equation 25] for $\alpha=0$ on the other hand results in

$\left\langle v_{\ell}^{2}\right\rangle-\frac{v}{\bar{v}_{\text {out }}} \sigma_{r}^{2}=\frac{v_{\mathrm{c}}^{2}}{3}$.

That is to say, if the rotation curve of the spherical halo is flat, the line-of-sight velocity dispersion is related to the circular velocity (and thus the mass) of the spherical halo such that $3\left\langle v_{\rho}^{2}\right\rangle \approx v_{c}^{2}$ (c.f., Lynden-Bell \& Frenk 1981; Evans et al. 1997; Wolf et al. 2010) independent of the behaviout 3 of $\beta$, to an extent that one can ignore the boundary term.

For more complicated mass profiles, an analytic result is in general difficult to obtain, except for some special cases. However, the special cases do include some interesting examples, one of which is the Hernquist halo profile traced by the populations with constant $\beta$, for which

$\frac{G M_{\mathrm{tot}}}{r_{0}}=\left\langle\left(\frac{32}{\pi} \frac{2-\beta}{4-3 \beta} \frac{R}{r_{0}}+6+\frac{8}{\pi} \frac{1-\beta}{2-\beta} \frac{r_{0}}{R}\right) v_{\ell}^{2}\right\rangle$

where $r_{0}$ is the scale length of the Hernquist halo. The proof is given in Appendix B together with further examples of mass profiles that result in a rational projected mass estimator.

Strictly speaking, the Hernquist halo cannot be traced by a population with $\beta>\frac{1}{2}$ at the centre in equilibrium since such a population must be cusped at the centre steeper than the dark halo cusp, which behaves as $\sim r^{-1}$ An \& Evans 2006). However, the constant anisotropy needs not extend to the centre and the mass estimate with varying anisotropy in the interval $(-\infty, 1]$ may be understood to be the range of the halo mass consistent with the observed line-of-sight velocity data set.

This projected mass estimator for the Hernquist profile is again notably consistent with the scale-free case of equation 25 at either extreme, $R \gg r_{0}$ (a finite total mass; $\alpha=1$ ) or $R \ll r_{0}$ ( $r^{-1}$ cusp; $\left.\alpha=-1\right)$. This indicates that if the tracers are restricted locally in the region where the halo potential can be approximated as a power law, equation 25 is a reasonable proxy for the mass estimator given proper boundary terms.

For general mass profiles, the weighting function can be derived numerically provided that $\lim _{r \rightarrow 0}(\mathrm{~d} \ln \tilde{\mu} / \mathrm{d} \ln r)<3$ (i.e., a cusped halo density profile). While the solution for the general case involves a double integral at the least, the function $w(R)$ for a few particular cases of constant $\beta$ with an analytic mass profile can be obtained through a simple quadrature (see Appendix A]. For example, the normalized weighting functions $\tilde{W}(\tilde{R})$ for the NFW profile - which replaces $\tilde{h}(\tilde{r})$ in eq. 20 together with the change to the line-of-sight velocity and $\tilde{R}=R / r_{0}$ - for some constant $\beta$ are provided in figure 1 of Evans et al. (2010). For the particular case of the NFW profile, it is also possible to derive the power-series expansion of $\tilde{W}(\tilde{R})$ at $\tilde{R}=0$ analytically, and also its asymptotic behaviour towards $\tilde{R} \rightarrow \infty$. In particular, we find at $\tilde{R}=0$ that $\tilde{W}(\tilde{R}) \simeq \frac{16}{\pi} C_{\beta} \tilde{R}^{-1}+8+O(\tilde{R})$ where $C_{\beta}=(1-\beta) /(2-\beta)$ and towards $\tilde{R} \rightarrow \infty$, that $\tilde{W}(\tilde{R}) \sim \tilde{R} /[\ln (1+\tilde{R})-1] \sim \tilde{R} /(\ln \tilde{R})$.

Finally, the circular orbit model $(\beta=-\infty)$ has some special points of interest, which are discussed in Appendix C

\subsubsection{Cored Halo Profiles}

Equation (25) is invalid for $\alpha=-2$ (i.e., an homogeneous sphere of radius $r_{\text {out }}$ ) because, provided that $v_{\ell}^{2} \neq 0$ for $R=0$ (i.e., $\beta \neq-\infty$ ), the average $\left\langle v_{\ell}^{2} / R^{2}\right\rangle$ that extends to $R=0$ diverges. However, since $\hat{I}_{\alpha=-2, \beta>-\infty}$ also diverges, it actually leads to an indeterminate form. In fact, the formal solution for equation 23 with $\tilde{\mu}=\left(r / r_{\text {out }}\right)^{3}$ exists in that $w(R)=2(1-2 \beta) r_{\text {out }}^{3} \delta\left(R^{2}\right)$ where $\delta(x)$ is the Dirac delta - that is to say, letting $R^{\alpha} / \Gamma\left(\frac{\alpha}{2}+1\right) \rightarrow \delta\left(R^{2}\right)$ as $\alpha \rightarrow 2$.

\footnotetext{
${ }^{3}$ Eq. 27] is in fact valid even if $\beta$ varies radially. This is because $\left\langle v^{2}\right\rangle=$ $3\left\langle v_{\ell}^{2}\right\rangle$ for any spherical system independent of the behaviour of $\beta$, and therefore eq. (6) is equivalent to eq. 27] for the halo with a flat rotation curve.
} 
In practice, the average $\left\langle v_{\ell}^{2} \delta\left(R^{2}\right)\right\rangle$ is directly related to the tracernumber-weighted line-of-sight velocity dispersion along the central line of sight, $\sigma_{\mathrm{L}, 0}$, or,

$$
\left\langle v_{\ell}^{2} \delta\left(R^{2}\right)\right\rangle=\frac{2 \pi}{N_{\text {tot }}} \int \mathrm{d} r v \sigma_{r}^{2}=\frac{\pi}{N_{\text {tot }}} \Sigma_{0} \sigma_{\mathrm{L}, 0}^{2}
$$

where $\Sigma_{0}$ is the tracer column density along the central line of sight. Therefore, for $\alpha=-2$ (and $\beta \neq-\infty$ ), equation (25) is replaced by

$$
\frac{G M_{\text {out }}}{r_{\text {out }}}=2(1-2 \beta) \frac{\Sigma_{0} \sigma_{\mathrm{L}, 0}^{2}}{\bar{\Sigma}_{\text {out }}}-3 \varsigma^{2}
$$

where $\bar{\Sigma}_{\text {out }}=N_{\text {tot }} /\left(\pi r_{\text {out }}^{2}\right)$ is the mean column density of the total tracer population.

It can also be shown that the properly derived weighting function $w(R)$ for any cored halo mass profile with $\lim _{r \rightarrow 0}(\mathrm{~d} \ln \tilde{\mu} / \mathrm{d} \ln r)=3$ contains the Dirac delta. Specifically, if $\lim _{r \rightarrow 0} r^{3} / \tilde{\mu}=L$ is a finite nonzero, then replacing $h$ with $\hat{h}=h-$ $2(1-2 \beta) L / r^{2}$ allows equation $(23)$ to be inverted. If $\hat{w}(R)$ is the solution for this inversion, the final weighting function that reproduces $\left\langle v_{r}^{2} h(r)\right\rangle=\left\langle v_{\ell}^{2} w(R)\right\rangle$ is found to be $w(R)=2(1-2 \beta) L \delta\left(R^{2}\right)+\hat{w}(R)$. That is to say, we find that

$\left\langle v_{\ell}^{2} \hat{w}(R)\right\rangle=\frac{G M_{\text {out }}}{r_{\text {out }}}-\frac{2(1-2 \beta) L}{r_{\text {out }}^{3}} \frac{\Sigma_{0} \sigma_{\mathrm{L}, 0}^{2}}{\bar{\Sigma}_{\text {out }}}+3 \varsigma^{2}$

given that

$\frac{1}{r}\left[\frac{r^{3} f}{\tilde{\mu}}-2(1-2 \beta) L\right]=\int_{0}^{r}\left(1-\beta \frac{R^{2}}{r^{2}}\right) \frac{\hat{w}(R) R \mathrm{~d} R}{\left(r^{2}-R^{2}\right)^{1 / 2}}$

and $L=\lim _{r \rightarrow 0} r^{3} / \tilde{\mu}$ is a finite constant (which further implies that $\lim _{r \rightarrow 0} r^{3} f / \tilde{\mu}=2(1-2 \beta) L$ and thus the above integral equation is invertible).

\section{THE SELF-CONSISTENT CASE}

If the tracer density $v(r)$ follows the same functional form as the dark halo density $\rho(r)$ (i.e., the mass-to-light is constant), then $\tilde{\mu}(r)$ is specified by the integral of $v(r)$ over the volume, and so the problem is completely determined. However, for this case the problem can be approached through a different simpler route, directly utilizing the fact that the potential and the tracer density are related through the Poisson equation. We find that the self-consistent case result in a formally identical mass estimator as the point-mass case except for an exact factor of two difference in the associated constant.

to

If $v / \rho$ is constant, using $\mathrm{d} M / \mathrm{d} r=4 \pi r^{2} \rho$, equation (2) reduces

$\frac{\mathrm{d} M^{2}}{\mathrm{~d} r}=-\frac{8 \pi}{G} \frac{r^{4}}{Q} \frac{\mathrm{d}}{\mathrm{d} r}\left(Q \rho \sigma_{r}^{2}\right)$.

Hence, integrating this on $r$ over $[0, \infty)$, we find that

$$
\frac{G M_{\mathrm{tot}}^{2}}{8 \pi}=-\int_{0}^{\infty} \frac{r^{4}}{Q} \frac{\mathrm{d}}{\mathrm{d} r}\left(Q \rho \sigma_{r}^{2}\right) d r=\int_{0}^{\infty} Q \rho \sigma_{r}^{2} \frac{\mathrm{d}}{\mathrm{d} r}\left(\frac{r^{4}}{Q}\right) \mathrm{d} r
$$

where $M_{\mathrm{tot}}=M(\infty)$ is the total mass. That is to say,

$M_{\mathrm{tot}}=\frac{2}{G}\left\langle(4-2 \beta) v_{r}^{2} r\right\rangle$.

The result is valid for an arbitrary functional form for the anisotropy parameter $\beta=\beta(r)$, but if $\beta$ is constant, this results in

$\left\langle v_{r}^{2} r\right\rangle=\frac{G M_{\mathrm{tot}}}{4(2-\beta)}$, which differs from the point-mass case in equation 16 by an exact factor of two. If the line-of-sight velocity dispersion is used instead, we find

$M_{\mathrm{tot}}=\frac{12}{G}\left\langle\frac{2-\beta}{3-2 \beta} v_{\ell}^{2} r\right\rangle$

in place of equation (22).

The calculation for the weighting function suitable for the projected separation as a observable is essentially identical to that found in Sect.4.2 as we would like to find the weighting function $w(R)$ satisfying $\left\langle v_{\ell}^{2} w(R)\right\rangle=\left\langle(4-2 \beta) v_{r}^{2} r\right\rangle$, which is to be substituted in equation 32 . This results in the identical integral equation (23) with $h=2(2-\beta) r$ or equivalently $\tilde{\mu}=1$. However, because of an additional factor of two in equation (32), the projected mass estimator for the self-consistent system with constant $\beta$ is different from equation (26) again exactly by a factor of two, i.e.,

$M_{\mathrm{tot}}=\frac{64}{\pi} \frac{2-\beta}{4-3 \beta} \frac{\left\langle v_{\ell}^{2} R\right\rangle}{G}$,

which encompasses the result of Heisler, Tremaine \& Bahcall (1985).

\section{DISCUSSION AND CONCLUSIONS}

Here, we have developed the theory of mass estimators. We are motivated by instances in astrophysics in which we wish to estimate the mass of a dark halo from positions and velocities of tracers such as stars, globular clusters, and satellite galaxies. The data sets may then be true distances and radial velocities (as for estimating the mass of the Milky Way from its satellite galaxies) or may be projected distances and line-of-sight velocities (as for the local dwarf spheroidal galaxies). In either case, we wish to estimate the mass of the dark halo from the kinematics of the tracer population.

For a given halo density profile, there exists the optimal weighting of these kinematic data. We have shown how to find it for any given specific density law and different kinds of positional and velocity data. This means that the mass within any radius can be calculated as the weighted sum of positions and velocities. We have worked out the formulae explicitly for a number of important cases, including scale-free, Hernquist and NFW haloes.

Although we have concentrated on general theoretical developments in this paper, the performance of the particular scale-free estimators has been tested and was already reported in an earlier paper (Watkins et al. 2010). We have also verified that our estimators (including the NFW ones) work well against simulation data in which a variety of effects such as halo asphericity, late infall of accreted material and lack of virial equilibrium are present. Even though these are not taken into account explicitly in our estimators, none the less they fare well against simulation data (Deason et al. 2010; Evans et al. 2010). Finally, applications of our theory to estimate the masses of the Milky Way and Andromeda galaxies (Watkins et al. 2010) and the dwarf spheroidals (Evans et al. 2010) are presented elsewhere.

One notable feature of the mass estimator theories that have been developed here is that they do not explicitly depend on any properties of the density of the tracer population $v$. In fact, the mass estimator incorporates the information through the definition of the average, that is,

$\langle u(\boldsymbol{r}, \boldsymbol{v})\rangle \equiv \frac{1}{N_{\text {tot }}} \int \mathrm{d}^{3} \boldsymbol{r} \mathrm{d}^{3} \boldsymbol{v} f(\boldsymbol{r}, \boldsymbol{v}) u(\boldsymbol{r}, \boldsymbol{v})=\frac{1}{N_{\text {tot }}} \int \mathrm{d}^{3} \boldsymbol{r} v(\boldsymbol{r}) \bar{u}(\boldsymbol{r})$ 
where

$\bar{u}(\boldsymbol{r}) \equiv \frac{1}{v(\boldsymbol{r})} \int \mathrm{d}^{3} \boldsymbol{v} f(\boldsymbol{r}, \boldsymbol{v}) u(\boldsymbol{r}, \boldsymbol{v})$

$v(\boldsymbol{r}) \equiv \int \mathrm{d}^{3} \boldsymbol{v} f(\boldsymbol{r}, \boldsymbol{v}) ; \quad N_{\mathrm{tot}} \equiv \int \mathrm{d}^{3} \boldsymbol{r} \mathrm{d}^{3} \boldsymbol{v} f(\boldsymbol{r}, \boldsymbol{v})=\int \mathrm{d}^{3} \boldsymbol{r} v(\boldsymbol{r})$

and $f(\boldsymbol{r}, \boldsymbol{v})$ is the phase space distribution function. However, given that the sampling of the tracers is statistically random, this formal average is estimated by the sample mean,

$\langle u(\boldsymbol{r}, \boldsymbol{v})\rangle \approx \frac{1}{N} \sum_{i}^{N} u\left(\boldsymbol{r}_{i}, \boldsymbol{v}_{i}\right)$.

That is to say, the effect of the tracer density in our mass estimator theories is naturally accounted through the spatial frequency of sampled tracers, and leaves no explicit dependence on $v$ in the consequent formulae. In practice, the choice of the sampled tracers may not necessarily be random. If there is some compelling reason to suspect sampling bias and/or the specific selection function is known, the sample mean can be estimated using any additional weighting accounting or correcting for the selection bias. However, even if a mild selection bias were to be present, the mass estimator may reasonably be robust without any explicit correction. This would be the case if the spatial variation of the quantity to be averaged for the mass estimator is not strongly correlated with the variation of the tracer density itself.

The conventional way in which mass estimation is performed is via the Jeans equations. The procedure is normally as follows: first, an assumption is made as to the luminosity density of the tracer population; secondly, the data set of discrete velocities is binned and smoothed to give the variation of the line-of-sight velocity dispersion with radius; thirdly, an assumption as to anisotropy is made (often that the anisotropy parameter $\beta \approx 0$ ) so that the line-of-sight velocity dispersion can be converted to the radial velocity dispersion, and fourthly the spherical Jeans equation is used to relate the underlying potential, and hence the enclosed mass, to the behaviour of the stellar kinematics. It is worth emphasizing that the results obtained for the matter distribution are often not robust, as they depend not just on the luminosity profile and the second velocity moments, but also their gradients.

In some sense, the techniques in this paper discard the wealth of information contained in the observed data set by taking spatial integrals over the whole system. However, there can be a number of advantages of this approach, especially if the number of datapoints are limited. First, it guards against overinterpreting the data set which can often happen with use of the Jeans equations. Second, the binning of the data, and their subsequent smoothing, are not needed. This is actually a great help, as Jeans modelling requires derivatives of functions derived from the binned and smoothed data. Third, the mass estimators are simple, requiring only weighted sums of positions and velocities, as opposed to solution of (at best) an ordinary differential equation.

For these reasons, we expect using mass estimators for discrete data to be a viable alternative to Jeans modelling. In the limits of large numbers of datapoints, we expect the mass estimators and Jeans modelling to yield similarly answers. This is borne out by the calculations of Evans et al. (2010) for dwarf spheroidals where data sets of thousands of radial velocities are available. When the the number of tracer datapoints is small, as often happens for estimating halo masses from satellite galaxies, mass estimators are the technique of choice. The precious data need not be smoothed, and the estimates of the enclosed mass are robust. We hope our tech- niques to be widely used in these instances, as Jeans modelling is either too cumbersome or too elaborate.

\section{ACKNOWLEDGMENTS}

This work originated from the first author's two separate two-week visits (February and October 2010) to the IoA (Cambridge), which were in part supported by the National Natural Science Foundation of China (NSFC) Research Fund for International Young Scientists, as well as the IoA's visitor grant. The authors also thank Laura L. Watkins and Alis J. Deason for Monte Carlo tests concerning the performance of the mass estimators with the Hernquist and the NFW haloes. JHA is supported by the Chinese Academy of Sciences (CAS) Fellowships for Young International Scientists, Grant No.:2009Y2AJ7.

\section{REFERENCES}

An J. H., Evans N. W., 2006, ApJ, 642, 752

An J. H., Evans N. W., 2009, ApJ, 701, 1500

Bahcall J. N., Tremaine S., 1981, ApJ, 244, 805

Deason A. J., McCarthy I., Font A., Evans N. W., Frenk C.S., Belokurov V., Libeskind N., Theuns T., Crain R., 2010, MNRAS, submitted

Dehnen W., 1993, MNRAS, 265, 250

Evans N. W., An J. H., 2005, MNRAS, 492, 498

Evans N. W., An J., Deason A. J., 2010, ApJ, submitted

Evans N. W., Hafner R. M., de Zeeuw P. T., 1997, MNRAS, 286, 315

Evans N. W., Wilkinson M. I., Perrett K. M., Bridges T. J., 2003, ApJ, 583, 752

Heisler J., Tremaine S., Bahcall J. N., 1985, ApJ, 298, 8

Hernquist L., 1990, ApJ, 356, 359

Kulessa A. S., Lynden-Bell D., 1992, MNRAS, 255, 105

Limber D. N., Mathews W. G., 1960, ApJ, 132, 286

Little B., Tremaine S., 1987, ApJ, 320, 493

Lynden-Bell D., Frenk C. S., 1981, The Observatory, 101, 200

Navarro J. F., Frenk C. S., White S. D. M., 1995, MNRAS, 275, 720

Strigari L. E., Bullock J. S., Kaplinghat M., Simon J. D., Geha M., Willman B., Walker M. G., 2008, Nat, 454, 1096

Tremaine S., Richstone D. O., Byun Y.-I., Dressler A., Faber S. M., Grillmair C., Kormendy J., Lauer T. R., 1994, AJ, 107, 634

Tully R. B., Rizzi L., Dolphin A. E., Karachentsev I. D., Karachentseva V. E., Makarov D. I., Makarova L., Sakai S., Shaya E. J., 2006, AJ, 132, 729

Veltmann Ü.-I. K., 1979, AZh, 56, 976 (English translation in SvA, 23, 551)

Walker M. G., Mateo M., Olszewski E. W., Peñarrubia J., Evans N. W, Gilmore G., 2009, ApJ, 704, 1274

Watkins L. L., Evans N. W., An J. H., 2010, MNRAS, 406, 264

White S. D. M., 1981, MNRAS, 195, 1037

Wilkinson M. I., Evans N. W., 1999, MNRAS, 310, 645

Wolf J., Martinez G. D., Bullock J. S., Kaplinghat M., Geha M., Muñoz R. R., Simon J. D., Avedo F. F., 2010, MNRAS, 406, 1220 


\section{APPENDIX A: HOW DO WE INVERT FOR THE PROJECTION WEIGHTING FUNCTION?}

First, let us rearrange equation (23) into an equivalent form;

$r^{3} h=\frac{f r^{4}}{\tilde{\mu}}=\int_{0}^{r}\left(r^{2}-R^{2}\right)^{1 / 2} w(R) R \mathrm{~d} R+(1-\beta) G(r)$

where

$G(r) \equiv \int_{0}^{r} \frac{w(R) R^{3} \mathrm{~d} R}{\left(r^{2}-R^{2}\right)^{1 / 2}}$.

However, we find that

$\frac{\mathrm{d}}{\mathrm{d} r}\left[\frac{1}{r} \int_{0}^{r}\left(r^{2}-R^{2}\right)^{1 / 2} w(R) R \mathrm{~d} R\right]=\frac{G(r)}{r^{2}}$.

Therefore, differentiating equation A1 after dividing it by $r$ leads to a differential equation for $G(r)$;

$(1-\beta) \frac{\mathrm{d} G}{\mathrm{~d} r}+\left(\frac{\beta}{r}-\frac{\mathrm{d} \beta}{\mathrm{d} r}\right) G(r)=r \frac{\mathrm{d}}{\mathrm{d} r}\left(\frac{f r^{3}}{\tilde{\mu}}\right)$.

Given $\tilde{\mu}(r)$ and $\beta(r)$, it is straightforward to solve equation (A4) numerically with the boundary condition $G(0)=0$. Furthermore, equation (A4) can be brought to an exact form

$\frac{\mathrm{d}}{\mathrm{d} r}(Q G)=\frac{r Q}{1-\beta} \frac{\mathrm{d}}{\mathrm{d} r}\left(\frac{f r^{3}}{\tilde{\mu}}\right)$,

by means of the integrating factor $Q(r)$ satisfying

$\frac{\mathrm{d} \ln Q}{\mathrm{~d} r}=-\frac{r}{1-\beta} \frac{\mathrm{d}}{\mathrm{d} r}\left(\frac{\beta}{r}\right)$.

Hence, if $Q(r)$ can be found, it is even possible to bring $G(r)$ to a quadrature. Finally, once $G(r)$ is found by some means, the weighting function $w(R)$ can be obtained though the inverse Abel transformation of equation $\mathrm{A} 2$, i.e.,

$w(R)=\frac{2}{\pi R^{2}} \int_{0}^{R} \frac{\mathrm{d} r}{\left(R^{2}-r^{2}\right)^{1 / 2}} \frac{\mathrm{d} G}{\mathrm{~d} r}$.

As an example, if $\beta$ is a constant, then $Q=r^{s}$ where $s=$ $\beta /(1-\beta)$ and thus

$G(r)=\left.\frac{1}{1-\beta} \frac{1}{r^{s}} \int_{0}^{r} \mathrm{~d} \hat{r} \hat{r}^{s+1} \frac{\mathrm{d}}{\mathrm{d} r}\left(\frac{f r^{3}}{\tilde{\mu}}\right)\right|_{r=\hat{r}}$.

The weighting function $w(R)$ is then found to be

$w(R)=\frac{2}{\pi(1-\beta) R^{2}} \int_{0}^{R} \frac{r \mathrm{~d} r}{\left(R^{2}-r^{2}\right)^{1 / 2}} \frac{\mathrm{d}^{2}}{\mathrm{~d} r^{2}}\left[\frac{1}{r^{s}} \int_{0}^{r} \frac{f(\hat{r}) \hat{r}^{3+s} \mathrm{~d} \hat{r}}{\tilde{\mu}(\hat{r})}\right]$.

For the isotropic case $(\beta=0)$, this simplifies to

$w(R)=\frac{2}{\pi R^{2}} \int_{0}^{R} \frac{r \mathrm{~d} r}{\left(R^{2}-r^{2}\right)^{1 / 2}} \frac{\mathrm{d}}{\mathrm{d} r}\left[(3+\hat{\alpha}) \frac{r^{3}}{\tilde{\mu}}\right]$

where $\hat{\alpha}=1-(\mathrm{d} \ln \tilde{\mu} / \mathrm{d} \ln r)$. Similarly for $\beta=\frac{1}{2}$ (i.e., $\left.s=1\right)$, the weighting function is found to be

$w(R)=\frac{4}{\pi R^{4}} \int_{0}^{R} \frac{r^{3} \mathrm{~d} r}{\left(R^{2}-r^{2}\right)^{1 / 2}} \frac{\mathrm{d}}{\mathrm{d} r}\left[(2+\hat{\alpha}) \frac{r^{3}}{\tilde{\mu}}\right]$.

For a system with purely radial orbits $(\beta=1)$, equation $\mathrm{A5}$ and those derived from it are not valid. However, it is still possible to solve for $w(R)$ from equation (23) or (A1), which result in

$w(R)=\frac{2}{\pi R^{2}} \int_{0}^{R} \frac{r \mathrm{~d} r}{\left(R^{2}-r^{2}\right)^{1 / 2}} \frac{\mathrm{d}^{2}}{\mathrm{~d} r^{2}}\left[(1+\hat{\alpha}) \frac{r^{4}}{\tilde{\mu}}\right]$.

The result for the purely circular orbit cases $(\beta=-\infty)$ on the other hand is obtained by inverting equation (C3) such that

$w(R)=\frac{4}{\pi R^{2}} \int_{0}^{R} \frac{\mathrm{d} r}{\left(R^{2}-r^{2}\right)^{1 / 2}} \frac{\mathrm{d}}{\mathrm{d} r}\left(\frac{r^{4}}{\tilde{\mu}}\right)$.

\section{APPENDIX B: THE PROJECTED MASS ESTIMATOR FOR THE HERNQUIST HALO}

Let us think of

$$
\begin{aligned}
J & =\left\langle\left(\frac{16}{\pi} \frac{2-\beta}{4-3 \beta} \frac{R}{r_{0}}+3+\frac{4}{\pi} \frac{1-\beta}{2-\beta} \frac{r_{0}}{R}\right) v_{\ell}^{2}\right\rangle \\
& =\frac{4 \pi}{N_{\text {tot }}} \int_{0}^{\infty} \mathrm{d} r r^{2} v \sigma_{r}^{2}\left(\frac{16}{\pi} \frac{2-\beta}{4-3 \beta} \frac{r}{r_{0}} \mathcal{S}_{2}+3 \mathcal{S}_{1}+\frac{4}{\pi} \frac{1-\beta}{2-\beta} \frac{r_{0}}{r} \mathcal{S}_{0}\right)
\end{aligned}
$$

where

$\mathcal{S}_{n}=\int_{0}^{\pi / 2} \mathrm{~d} \theta \sin ^{n} \theta\left(1-\beta \sin ^{2} \theta\right)$.

We find that

$\mathcal{S}_{2}=\frac{\pi}{16}(4-3 \beta) ; \quad \mathcal{S}_{1}=1-\frac{2}{3} \beta ; \quad \mathcal{S}_{0}=\frac{\pi}{4}(2-\beta)$.

Next,

$$
\begin{aligned}
\frac{16}{\pi} \frac{2-\beta}{4-3 \beta} \frac{r}{r_{0}} \mathcal{S}_{2}+3 \mathcal{S}_{1}+\frac{4}{\pi} \frac{1-\beta}{2-\beta} & \frac{r_{0}}{r} \mathcal{S}_{0} \\
& =\frac{1}{2 r_{0} r^{2-2 \beta}} \frac{\mathrm{d}}{\mathrm{d} r}\left[r^{2-2 \beta}\left(r_{0}+r\right)^{2}\right] .
\end{aligned}
$$

Therefore

$$
\begin{aligned}
J & =\frac{2 \pi}{r_{0} N_{\text {tot }}} \int_{0}^{\infty} d r r^{2 \beta} v \sigma_{r}^{2} \frac{\mathrm{d}}{\mathrm{d} r}\left[r^{2-2 \beta}\left(r_{0}+r\right)^{2}\right] \\
& =-\frac{2 \pi}{r_{0} N_{\text {tot }}} \int_{0}^{\infty} d r r^{2-2 \beta}\left(r_{0}+r\right)^{2} \frac{\mathrm{d}}{\mathrm{d} r}\left(r^{2 \beta} v \sigma_{r}^{2}\right) .
\end{aligned}
$$

With the spherical Jeans equation for a constant $\beta$ (eq. 2. with $Q=$ $r^{2 \beta}$ ) and the mass profile for the Hernquist halo (eq. 17 with $\gamma=$ $p=1$ ), we find that

$J=\frac{2 \pi}{r_{0} N_{\mathrm{tot}}} \int_{0}^{\infty} \mathrm{d} r r^{2} v G M_{\mathrm{tot}}=\frac{G M_{\mathrm{tot}}}{2 r_{0}}$.

Similar calculations can also demonstrate the existence of a rational projected mass estimator for the particular mass models in equation (17) such that

$\frac{G M_{\mathrm{tot}}}{r_{0}}=\left\langle\left(\frac{32}{\pi} \frac{2-\beta}{4-3 \beta} \frac{R}{r_{0}}+3\right) v_{\ell}^{2}\right\rangle$

for the Jaffe model

$\rho(r)=\frac{M_{\mathrm{tot}}}{4 \pi} \frac{r_{0}}{r^{2}\left(r_{0}+r\right)^{2}} ; \quad \frac{M(r)}{M_{\mathrm{tot}}}=\frac{r}{r_{0}+r}$,

and

$\frac{G M_{\mathrm{tot}}}{r_{0}}=\left\langle\left(\frac{32}{\pi} \frac{2-\beta}{4-3 \beta} \frac{R}{r_{0}}+\frac{8}{\pi} \frac{1-\beta}{2-\beta} \frac{r_{0}}{R}\right) v_{\ell}^{2}\right\rangle$

for

$\rho(r)=\frac{M_{\mathrm{tot}}}{2 \pi} \frac{r_{0}^{2}}{r\left(r_{0}^{2}+r^{2}\right)^{2}} ; \quad \frac{M(r)}{M_{\mathrm{tot}}}=\frac{r^{2}}{r_{0}^{2}+r^{2}}$.

An analytic example for the cored models that require the Dirac delta in the weighting function is found for

$\rho(r)=\frac{3 M_{\mathrm{tot}}}{4 \pi} \frac{r_{0}}{\left(r_{0}+r\right)^{4}} ; \quad \frac{M(r)}{M_{\mathrm{tot}}}=\left(\frac{r}{r_{0}+r}\right)^{3}$

whose formal form of the projected mass estimator is given by

$\frac{G M_{\mathrm{tot}}}{r_{0}}=\left\langle\left[\frac{32}{\pi} \frac{2-\beta}{4-3 \beta} \frac{R}{r_{0}}+9+\frac{24}{\pi} \frac{1-\beta}{2-\beta} \frac{r_{0}}{R}+2(1-2 \beta) r_{0}^{2} \delta\left(R^{2}\right)\right] v_{\ell}^{2}\right\rangle$. 


\section{APPENDIX C: PURELY CIRCULAR ORBITS}

For an extreme scenario, one can imagine that all tracers are in circular orbits and their orbital phases and orientations are completely random (hence there is no net angular momentum of tracer populations in each shell of a fixed radius). This corresponds to the case that $\beta=-\infty$ everywhere. Since $\sigma_{r}^{2}=0$ everywhere, the $v_{r}$-based mass estimator is invalid for this case, but the line-of-sight velocity based ones are still applicable. Although the final result turns out to be the same as the simple limit to $\beta \rightarrow-\infty$, we can derive them via physically consistent routes.

Let us start by noting that the line-of-sight velocity dispersion of the population in purely circular orbits with random orientations is given by

$\sigma_{\ell}^{2}=\frac{v_{\mathrm{c}}^{2}}{2} \sin ^{2} \theta=\frac{G M(r)}{2 r} \sin ^{2} \theta$

where $v_{\mathrm{c}}$ is the circular speed of the spherical halo at $r$. First, we consider the case that the radial distances $r$ of the individual tracers to the halo centre are known. Then, the average of $v_{\ell}^{2}$ weighted by a function $h(r)$ is found to be

$$
\begin{aligned}
\left\langle v_{\ell}^{2} h(r)\right\rangle & =\frac{4 \pi}{N_{\text {tot }}} \int_{r_{\text {in }}}^{r_{\text {out }}} \mathrm{d} r \int_{0}^{\pi / 2} \mathrm{~d} \theta r^{2} \sin \theta v \sigma_{\ell}^{2} h(r) \\
& =\frac{4 \pi}{N_{\text {tot }}} \int_{r_{\text {in }}}^{r_{\text {out }}} \mathrm{d} r r^{2} v \frac{v_{\mathrm{c}}^{2} h}{2} \int_{0}^{\pi / 2} \mathrm{~d} \theta \sin ^{3} \theta \\
& =\frac{4 \pi}{N_{\text {tot }}} \int_{r_{\text {in }}}^{r_{\text {out }}} \mathrm{d} r r^{2} v \frac{G M h}{3 r} .
\end{aligned}
$$

Hence, if one chooses $h=3 r / \tilde{\mu}$ where $\tilde{\mu}(r)$ is the assumed mass profile (eq. 12), the total mass $M_{\text {out }}$ can be isolated by

$M_{\mathrm{out}}=\frac{3}{G}\left\langle\frac{v_{\ell}^{2} r}{\tilde{\mu}}\right\rangle$,

which is consistent with equation (22) in the limit of $\beta \rightarrow-\infty$. The result does not involve the boundary term because $\sigma_{r}^{2}=0$ everywhere for the assumed system.

For the case that only the projected distances $R$ to the halo centre are available, we consider the similar weighted average of $v_{\ell}^{2}$ as Sect.4.2 that is,

$\left\langle v_{\ell}^{2} w(R)\right\rangle=\frac{4 \pi}{N_{\mathrm{tot}}} \int \mathrm{d} r r^{2} v \frac{G M}{2 r} \int_{0}^{\pi / 2} \mathrm{~d} \theta \sin ^{3} \theta w(R)$.

Hence, if $w(R)$ is chosen to satisfy the integral equation

$\frac{2 r^{4}}{\tilde{\mu}}=\int_{0}^{r} \frac{w(R) R^{3} \mathrm{~d} R}{\left(r^{2}-R^{2}\right)^{1 / 2}} \equiv G(r)$,

the total mass is related to the weighted average via $G M_{\text {out }}=$ $\left\langle v_{\ell}^{2} w(R)\right\rangle$. For the scale-free case (eq. [14), we have $w(R) \propto R^{\alpha}$ and therefore

$M_{\mathrm{out}}=\frac{4 \Gamma\left(\frac{\alpha+5}{2}\right)}{\pi^{1 / 2} \Gamma\left(\frac{\alpha}{2}+2\right)} \frac{\left\langle v_{\ell}^{2} R^{\alpha}\right\rangle}{G r_{\mathrm{out}}^{\alpha-1}}$,

which is valid for $\alpha>-4$. For a central point mass ( $\alpha=1$ ), this becomes $G M_{\mathrm{tot}}=\frac{32}{3 \pi}\left\langle v_{\ell}^{2} R\right\rangle$. That is to say, the mass estimate under the assumption that the tracers in purely circular orbits is smaller by a factor of three and 1.5 respective compared to the case that they are in the radial orbits or the isotropic case (see eq. 26).

The calculations for the self-consistent case are similar. First, we use $v / N_{\text {tot }}=\rho / M_{\text {tot }}$ (assuming $r_{\text {in }}=0$ and $r_{\text {out }}=\infty$ ) and $\mathrm{d} M / \mathrm{d} r=4 \pi r^{2} \rho$ to further reduce equation (C1) to

$\left\langle v_{\ell}^{2} h(r)\right\rangle=\frac{1}{M_{\mathrm{tot}}} \int_{0}^{\infty} \mathrm{d} r \frac{G h}{6 r} \frac{\mathrm{d} M^{2}}{\mathrm{~d} r}$.
With $h=6 r$, we find that $M_{\text {tot }}=6 G^{-1}\left\langle v_{\ell}^{2} r\right\rangle$, which differs from equation (C2) with $\tilde{\mu}=1$ by a factor of two and is also the limit of equation (33) as $\beta \rightarrow-\infty$. With $R$ as an observable instead of $r$, we have $G(r)=4 r^{4}$ in place of equation (C3), which ultimately leads to the mass estimator, $G M_{\mathrm{tot}}=\frac{64}{3 \pi}\left\langle v_{\ell}^{2} R\right\rangle$, which is the same as equation (34) for $\beta \rightarrow-\infty$. 